\title{
Selección de un Modelo Primario para Describir la Curva de Crecimiento de Bacterias Lácticas y Brochothrix thermosphacta sobre Emulsiones Cárnicas Cocidas
}

María E. Cayré (1), Graciela M. Vignolo (2) y Oscar A. Garro (1)

(1) Facultad de Agroindustrias, Universidad Nacional del Nordeste, Comandante Fernández 755

(3700). Presidencia Roque Sáenz Peña, Chaco-Argentina.

(2) Centro de Referencia para Lactobacilos (CERELA), Chacabuco 145 (3400),

San Miguel de Tucumán, Tucumán-Argentina (e-mail: ecayre@fai.unne.edu.ar)

\begin{abstract}
Resumen
Se han comparado tres modelos primarios para describir la curva de crecimiento de bacterias lácticas y Brochothrix thermosphacta sobre emulsiones cárnicas cocidas y para estimar los parámetros cinéticos de crecimiento: tiempo de latencia $(\lambda)$, máxima velocidad específica de crecimiento $\left(\mu_{\max }\right) \mathrm{y}$ máxima densidad bacteriana $\left(y_{\max }\right)$. Se usaron los modelos Logístico, Gompertz y Baranyi y se comparó la bondad de ajuste de los modelos, la incertidumbre y precisión de las estimaciones. Los modelos de Gompertz y Baranyi produjeron los mejores ajustes en la mayoría de los casos presentados. No se detectaron diferencias apreciables entre los modelos en relación a la incertidumbre y precisión de las estimaciones de $y_{\max }$ y $\mu_{\max }$. Sin embargo, las estimaciones de $\lambda$ dadas por el modelo de Baranyi fueron menos exactas y los resultados muestran que el modelo de Gompertz mostró un mejor comportamiento.
\end{abstract}

Palabras claves: curva de crecimiento, bacterias lácticas, Brochothrix thermosphacta, modelado,

\section{Selection of a Primary Model for to Describe the Growth Curve of Lactic Acid Bacteria and Brochothrix thermosphacta on Cooked Meat Emulsions}

\begin{abstract}
Three primary models to describe the growth curve of lactic acid bacteria and Brochothrix thermosphacta on cooked meat emulsions have been compared. The following kinetic growth parameters were determined: lag time $(\lambda)$, maximum specific growth rate $\left(\mu_{\max }\right)$ and maximum microbial density $\left(y_{\max }\right)$. The models Logístico, Gompertz y Baranyi were employed and the goodness-of-fit of the models, the uncertainty and the precision of the estimations were compared. The Gompertz and the Baranyi models gave the best fit in most cases. No appreciable differences were detected between the models regarding to the uncertainty and precision of the estimations of $y_{\max }$ and $\mu_{\max }$. Nevertheless, the estimations of $\lambda$ given by the Baranyi model were less accurate and the results show that the Gompertz model showed a better behavior.
\end{abstract}

Keywords: growth curve, lactic acid bacteria, Brochothrix thermosphacta, modeling 


\section{INTRODUCCIÓN}

El objetivo de un modelo primario es describir matemáticamente la curva de crecimiento generada por los microorganismos de interés bajo condiciones ambientales definidas con el objeto de estimar los parámetros cinéticos que caracterizan dicha curva: tiempo de latencia, máxima velocidad específica de crecimiento y máxima densidad celular. Con el objeto de estimar los parámetros cinéticos de crecimiento de forma objetiva, desde 1980 se han propuesto una serie de modelos matemáticos. Baty y Delignette-Müller (2004) clasificaron a los modelos primarios como: modelos sigmoidales, entre los que se encuentran las versiones modificadas de los modelos Logístico y de Gompertz; modelos con una función de ajuste, como los propuestos por Baranyi y Roberts (1994) y Buchanan et al., (1997) y modelos de compartimentos como el propuesto por Hills y Wrigth (1994).

La propiedad más importante de un modelo primario es que describa de forma adecuada el crecimiento de los microorganismos y permita obtener estimaciones precisas de los parámetros que caracterizan dicho crecimiento. La variabilidad de las estimaciones depende de la técnica usada para monitorear el crecimiento y del modelo usado (Dalgaard y Koutsoumanis, 2001; Baty et al., 2002). Dada la existencia de diferentes modelos primarios, se hace necesario comparar el comportamiento de los mismos a fin de seleccionar el que asegure mejores resultados para un conjunto determinado de datos. McMeekin et al. (1993) indicaron que, aspectos tales como la bondad de ajustes y las propiedades de estimación deben ser tenidos en cuenta en la comparación de modelos competidores. Baty y Delignette-Müller (2004) sugirieron que las variaciones inter-modelo deben ser consideradas. En este trabajo se compararon las propiedades de ajuste y de las estimaciones dadas por diferentes modelos de crecimiento a fin de seleccionar el más adecuado para describir el crecimiento de bacterias lácticas y Brochothrix termos-phacta alterantes comunes de emulsiones cárnicas cocidas.

\section{MATERIALES Y MÉTODOS}

\section{Datos Experimentales}

Se trabajó con un conjunto de datos correspondientes a la generación experimental de 25 curvas de crecimiento, 12 de las cuales corresponden a bacterias lácticas y 13 a $B$. thermosphacta. Los datos corresponden al crecimiento de ambos microorganismos sobre salchichas de Viena elaboradas por una industria local. Las muestras naturalmente contaminadas fueron envasadas al vacío y almacenadas a diferentes temperaturas de refrigeración. El crecimiento se monitoreó mediante recuento en placa de viables. Los recuentos de bacterias lácticas totales (BAL) se obtuvieron sobre agar MRS (Merck, Alemania) después de incubar las placas a $30^{\circ} \mathrm{C}$ por 72 horas, mientras que $B$. thermosphacta fue enumerada sobre agar STAA con suplementos selectivos (Oxoid, Inglaterra) después de 48 horas de incubación a $25^{\circ} \mathrm{C}$. Los recuentos se expresaron como ln (ufc $\mathrm{g}^{-1}$ ).

\section{Modelos Matemáticos}

En función de la simplicidad y frecuencia de uso se seleccionaron tres modelos matemáticos para la comparación. Se usaron las versiones modificadas de los modelos Logístico y de Gompertz propuestas por Zwietering et al. (1990) y el modelo de Baranyi (Baranyi y Roberts, 1994). Las expresiones matemáticas de estos modelos son:

Modelo Logístico

$$
y(t)=y_{0}+\frac{y_{\max }-y_{0}}{\left\{1+\exp \left[\frac{4 \mu_{\max }}{y_{\max }-y_{0}}(\lambda-t)+2\right]\right\}}
$$

Modelo de Gompertz

$$
\mathrm{y}(\mathrm{t})=\mathrm{y}_{0}+\left(\mathrm{y}_{\max }-\mathrm{y}_{0}\right) \exp \left\{-\exp \left[1+\mu_{\max } \cdot \mathrm{e}\left(\frac{\lambda-\mathrm{t}}{\mathrm{y}_{\max }-\mathrm{y}_{0}}\right)\right]\right\}
$$


Modelo de Baranyi

$y(t)=y_{\max }+\ln \left[\frac{-1+\exp \left(\mu_{\max } \cdot \lambda\right)+\exp \left(\mu_{\max } \cdot t\right)}{-1+\exp \left(\mu_{\max } \cdot t\right)+\exp \left(\mu_{\max } \cdot \lambda+y_{\max }-y_{0}\right)}\right]$

Donde:

$y(t)=\ln N(t)$, siendo $N(t)$ la densidad bacteriana (ufc. $g^{-1}$ ) al tiempo $t$.

$y_{0}=\ln N_{0}$, siendo $N_{0}$ el valor asintótico inferior y aproximadamente igual a la densidad bacteriana inicial $\left(\right.$ ufc. $\left.g^{-1}\right)$.

$y_{\max }=\ln N_{\max }$, siendo $N_{\max }$ el valor asintótico superior y aproximadamente igual a la máxima densidad bacteriana (ufc. g $^{-1}$ ).

$\mu_{\max }=$ máxima velocidad específica de crecimiento $\left(\right.$ tiempo $\left.^{-1}\right)$.

$\lambda=$ tiempo de latencia (tiempo).

\section{Ajuste de los Modelos Matemáticos}

Los modelos seleccionados se ajustaron a los datos experimentales mediante el algoritmo de Marquardt en STATGRAPHICS Plus Versión 4.0 (Statistical Graphics Corp., MD 20852, Estados Unidos). En todos los casos, los valores de $y_{0}$ corresponden a los valores medios obtenidos experimentalmente.

\section{Comparación de los Modelos}

La bondad de ajuste de los modelos se evaluó mediante los valores del coeficiente de determinación $\left(R^{2}\right)$ y la raíz cuadrada de los cuadrados medios del error (RMSE) de cada ajuste. Cuánto más cercano a 1 es el valor de $\mathrm{R}^{2}$ y más bajo el valor de RMSE, mejor ajusta el modelo.

Para cada estimación dada por los modelos se calculó el coeficiente de variación asociado a dicha estimación mediante la siguiente expresión:

$C V_{E}=\left(S E_{E} / E\right) \cdot 100$

donde $\mathrm{SE}_{\mathrm{E}}$ es el error estándar de la estimación y $\mathrm{E}$ es le valor estimado.

También se calcularon, para cada parámetro estimado, los coeficientes de variación inter-modelo como:

$\mathrm{CV}_{\text {inter-modelo }}=\left(\mathrm{S}_{\mathrm{E}} / \overline{\mathrm{E}}\right) \cdot 100$

donde $S_{E}$ y $\bar{E}$ son la desviación estándar y la media aritmética de las estimaciones, respectivamente.

Los valores de los parámetros estimados por cada uno de los modelos se compararon a través de las relaciones $R\left(E_{G} / E_{L}\right), R\left(E_{G} / E_{B}\right)$ y $R\left(E_{L} / E_{B}\right)$, donde $E$ corresponde al valor estimado de los parámetros por los modelos Logístico (L), de Gompertz $(G)$ y de Baranyi $(B)$. Para cada modelo y para cada parámetro se determinaron la media aritmética y la desviación estándar de las relaciones $\mathrm{R}$. La precisión de las estimaciones dadas por los diferentes modelos se compararon mediante sus intervalos de confianza asintóticos al $95 \%$ de confianza. Los intervalos fueron expresados como porcentaje del valor estimado.

\section{RESULTADOS Y DISCUSIÓN}

Los recuentos bacterianos obtenidos experimentalmente se usaron para ajustar los modelos matemáticos de crecimiento. En términos generales, los tres modelos dan visualmente buenos ajustes. Ejemplos de los ajustes se muestran en las Figuras 1 a y b para BAL y $B$. thermosphacta respectivamente. Para los ajustes realizados, el modelo de Gompertz produce los valores más bajos de MRSE en el $48 \%$ de los casos presentados, el modelo de Baranyi en el $44 \%$ y el modelo Logístico sólo en el $8 \%$ de los casos. 
La comparación de los valores de MRSE de los modelos de Gompertz y Logístico, mostró que en la mayoría de los casos, el modelo de Gompertz es más adecuado para la descripción de los datos. Estos resultados son coincidentes con los reportados por Gibson et al., (1987) sugirieron que la falta de simetría de algunas curvas explicaría las mayores desviaciones entre observaciones y predicciones obtenidas con el modelo Logístico puesto que éste es una función simétrica respecto al punto de máxima velocidad de crecimiento mientras que el modelo de Gompertz no lo es.

Comparaciones de la bondad de ajuste realizadas entre los modelos de Gompertz y Baranyi indican una preferencia del modelo de Baranyi sobre el de Gompertz con diferencias promedio de un $13 \%$ hasta un $43 \%$ entre los valores de MRSE (Buchanan et al., 1997; Baty y Delignette-Müller, 2004).Para los datos analizados en este trabajo, la preferencia de un modelo sobre el otro no es tan evidente. En efecto, para 12 de los 25 casos presentados el modelo de Gompertz produce los valores más bajos de MRSE y, en promedio, los valores correspondientes al modelo de Baranyi fueron un $12,5 \%$ más altos. Para los 13 casos restantes, los valores de MRSE producidos por el modelo de Gompertz fueron un $17 \%$ más altos que los correspondientes al modelo de Baranyi, lo que evidencia un comportamiento similar de ambos modelos.
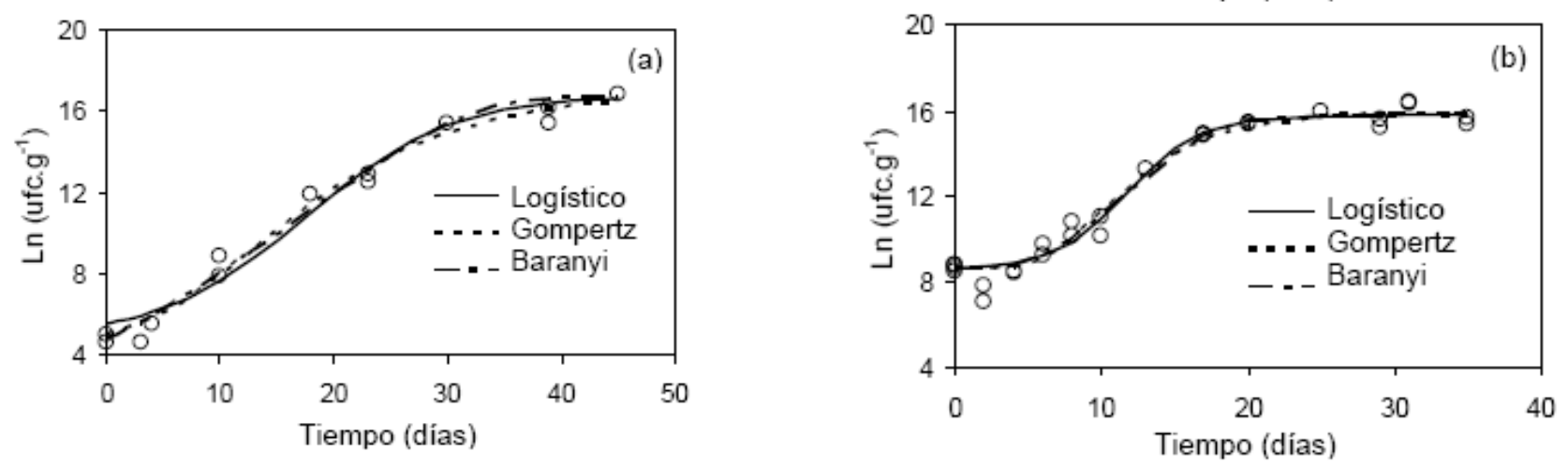

Fig. 1. Ajuste de los modelos primarios a los recuentos de (a) BAL y (b) B. thermosphacta

Los valores de $\mathrm{R}^{2}$ obtenidos en todos los ajustes variaron entre un mínimo de 0,904 y un máximo de 0,998 . En el $75 \%$ de los casos, los valores fueron mayores a 0,970. Estos resultados indican que los tres modelos explican un alto porcentaje de la variabilidad de los logaritmos de los recuentos bacterianos en el tiempo.

Los valores más altos de $\mathrm{R}^{2}$ fueron obtenidos con los modelos de Gompertz y Baranyi. Sólo en 2 de los 25 casos presentados el modelo Logístico produjo valores de $R^{2}$ más altos que los otros modelos. En la Tabla 1 se muestran los valores medios y las desviaciones estándar de las relaciones $R$ entre las estimaciones (E) de los parámetros cinéticos de crecimiento dadas por los diferentes modelos. Los coeficientes de variación medios asociados a las estimaciones de los modelos y los coeficientes inter-modelo se resumen en la Tabla 2.

La Fig. 2 muestra los diagramas de caja y bigote para los intervalos de confianza asintóticos expresados como porcentaje de los valores estimados de los parámetros cinéticos de crecimiento por los diferentes modelos.

Los valores cercanos a la unidad de las relaciones $R$ para las estimaciones de y el coeficiente variación medio intermodelo indican que los tres modelos dan valores similares para este parámetro. Los coeficientes de variación asociados a estas estimaciones son todos inferiores al $5 \%$ lo que le confiere un bajo nivel de incertidumbre a las mismas. No se aprecian grandes diferencias en la precisión con que cada modelo estima el parámetro $y_{\max }$ (Fig. 2a). 
Tabla 1. Relaciones $R$ entre las estimaciones de los parámetros ( ${ }^{\mathrm{a} G o m p e r t z,}{ }^{\mathrm{b}}$ Logístico, ${ }^{\mathrm{c}}$ Baranyi, ${ }^{\mathrm{d}}$ Media aritmética, ${ }^{\mathrm{e}}$ Desviación estándar)

\begin{tabular}{|c|c|c|c|}
\hline \multirow{2}{*}{$E$} & \multicolumn{3}{|c|}{ Relaciones $\mathrm{R}$} \\
\cline { 2 - 4 } & $\mathrm{G}^{\mathrm{a}} / \mathrm{L}^{\mathrm{b}}$ & $\mathrm{G} / \mathrm{B}^{\mathrm{c}}$ & $\mathrm{L} / \mathrm{B}$ \\
\hline \multirow{2}{*}{$y_{\max }$} & $\begin{array}{c}1,007^{\mathrm{d}} \pm \\
0,010^{\mathrm{e}}\end{array}$ & $\begin{array}{c}1,009 \pm \\
0,010\end{array}$ & $\begin{array}{c}1,009 \pm \\
0,003\end{array}$ \\
\hline \multirow{3}{*}{$\mu_{\max }$} & $0,977 \pm$ & $1,208 \pm$ & $1,238 \pm$ \\
& 0,091 & 0,141 & 0,131 \\
\hline \multirow{2}{*}{$\lambda$} & $0,855 \pm$ & $1,501 \pm$ & $1,822 \pm$ \\
& 0,100 & 0,764 & 1,060 \\
\hline
\end{tabular}

Tabla 2. Coeficientes de variación medios asociados a las estimaciones ('Logístico, ${ }^{\mathrm{b}}$ Gompertz, ${ }^{\mathrm{c}}$ Baranyi, ${ }^{\mathrm{d}}$ Media aritmética)

\begin{tabular}{|c|c|c|c|c|}
\hline \multirow{2}{*}{$E$} & \multicolumn{4}{|c|}{ Coeficientes de Variación (\%) } \\
\cline { 2 - 5 } & $\mathrm{L}^{\mathrm{a}}$ & $\mathrm{G}^{\mathrm{b}}$ & $\mathrm{B}^{\mathrm{c}}$ & Inter-modelo \\
\hline$y_{\max }$ & $1,52^{\mathrm{d}}$ & 1,59 & 1,43 & 0,66 \\
\hline$\mu_{\max }$ & 13,47 & 12,40 & 11,23 & 10,75 \\
\hline$\lambda$ & 17,85 & 18,24 & 37,05 & 20,83 \\
\hline
\end{tabular}

Para las estimaciones de $\mu_{\max }$ las relaciones $\mathrm{R}$ muestran que los modelos Logístico y de Gompertz tienden a sobrestimar este parámetro respecto del modelo de Baranyi. Resultados similares fueron reportados por Augustin y Carlier (2000) y Perni et al., (2005). A su vez, las estimaciones del modelo Logístico son más altas que las del modelo de Gompertz. Sin embargo, los coeficientes de variación asociados a las estimaciones (Tabla 2) y la precisión de las mismas (Fig. 2b) evidencian pequeñas diferencias entre los modelos. Las diferencias entre los modelos se hacen más notorias al considerar las estimaciones de $\lambda$. También para este parámetro los modelos Logístico y de Gompertz dan estimaciones más altas que el modelo de Baranyi. Sin embargo, las diferencias son más marcadas que para $\mu_{\max }$.

Los coeficientes de variación asociados a las estimaciones de $\lambda$ (Tabla 2) y la amplitud de los intervalos de confianza (Fig. 2c) son mayores que para los otros parámetros. Varios autores enfatizaron sobre las dificultades en las estimaciones de la duración de la fase de latencia (McKellar, 1997; Baranyi, 2002; Baty y Delignette-Müller, 2004) debidas principalmente a la falta de entendimiento fisiológico del fenómeno de latencia.

Las mayores diferencias entre los modelos Logístico y de Gompertz con respecto al de Baranyi en relación a la incertidumbre y precisión de las estimaciones de $\lambda$ pueden explicarse en función de la cantidad de datos experimentales a lo largo de la fase de latencia. Grijspeerdt y Vanrolleghem (1999) y Swinnen et al. (2004) indicaron que para reducir la incertidumbre de las estimaciones dadas por el modelo de Baranyi se necesita un intenso muestreo en la etapa de transición entre la fase de latencia y la exponencial. También, Baty y Delignette- Müller (2004) y Poschet et al. (2004) demostraron que la imprecisión de las estimaciones de este modelo está claramente correlacionada con la cantidad de datos. Cuanto menor fue la cantidad de datos a lo largo de la fase de latencia, mayor fue la incertidumbre y menor la precisión de las estimaciones dadas por los tres modelos. Sin embargo, la influencia fue más pronunciada sobre las estimaciones dadas por el modelo de Baranyi lo cual queda reflejado en los coeficientes de variación (Tabla 2) y en la amplitud de los intervalos de confianza asintóticos (Fig. 2c).

\section{CONCLUSIONES}

En términos de bondad de ajuste puede concluirse que si bien, ninguno de los modelos produce el mejor ajuste para todos los casos, con mayor frecuencia los modelos de Gompertz y Baranyi producen los valores más bajos de MRSE y más altos de $R^{2}$ y resultan más adecuados que el modelo Logístico para la descripción de los datos experimentales.

En cuanto a las estimaciones de los parámetros cinéticos de crecimiento, no se evidenciaron diferencias considerables entre los modelos en relación a la incertidumbre y precisión de las estimaciones de $y_{\max } \mathrm{y} \mu_{\max }$. Para estos parámetros los coeficientes de variación inter-modelo son menores que los coeficientes de variación dentro de cada modelo y los intervalos de confianza de las estimaciones dadas son, en promedio, menores al $30 \%$ de los valores estimados. 

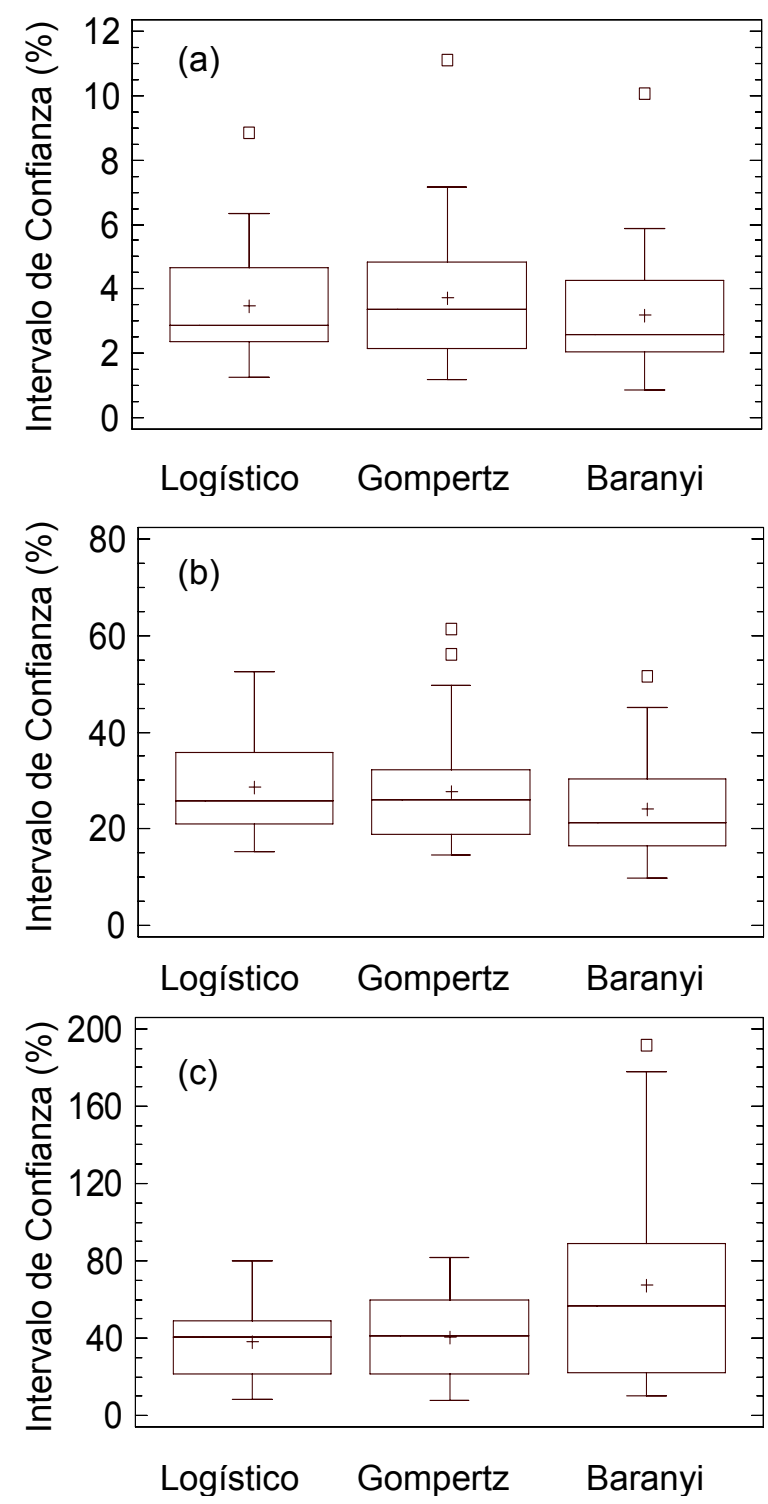

Fig. 2 Diagramas de caja y bigote para los intervalos de confianza de las estimaciones de (a) $y_{\max }$, (b) $\mu_{\max }$ y (c) $\lambda$ dadas por los modelos primarios.

Las mayores diferencias entre los modelos se manifiestan en las estimaciones de $\lambda$. Si bien los tres modelos tienen dificultades en la estimación de este parámetro para los datos presentados, el modelo de Baranyi produce estimaciones menos certeras y precisas que los modelos Logístico y de Gompertz. En función de la evaluación de las propiedades de ajuste y de las estimaciones dadas por los modelos, el modelo de Gompertz resultó más adecuado y fue seleccionado para la descripción de los datos experimentales presentados.

La estimación de los parámetros de crecimiento bajo diferentes condiciones ambientales, tales como temperatura, pueden utilizarse en la construcción de modelos que permitan predecir el crecimiento de microorganismos alterantes como bacterias lácticas y Brochothrix thermosphacta y estimar la vida útil del producto.

\section{AGRADECIMENTOS}

Los autores agradecen el financiamiento de la Secretaría General de Ciencia y Técnica de la Universidad Nacional del Nordeste y el Consejo Nacional de Investigaciones Científicas y Técnicas (CONICET), Argentina y la colaboración de la Dra. Marcela Castro. 


\section{REFERENCIAS}

Augustin, J.C. y V. Carlier. Mathematical modeling of the growth rate and lag time for Listeria monocytogenes. Int. J. Food Microbiol. 56 (1), 29-51 (2000).

Baranyi, J. Stochastic modeling of bacterial lag phase. Int. J. Food Microbiol. 73 (2-3), 203-206 (2002).

Baranyi, J. y T. A. Roberts. A dynamic approach to predicting the bacterial growth in food. Int. J. Food Microbiol. 23(3-4), 277-294 (1994).

Baty, A.S. y M.L. Delignette-Müller. Estimating the bacterial lag time: which model, which precision? Int. J. Food Microbiol. 91 (3), 261-277 (2004).

Baty, A.S., J.P. Flandrois y M.L. Delignette-Müller. Modeling the lag time of Listeria monocytogenes from viable count enumeration and optical density data. Appl. Environ. Microbiol. 68 (12), 5816-5825 (2002).

Buchanan, R.L., R.C. Whiting y W.C., Damert. When is simple good enough: a comparison of the Gompertz, Baranyi and three-phase linear models for fitting bacterial growth curves. Food Microbiol. 14 (4), 313-326 (1997).

Dalgaard, P. y K. Koutsoumanis. Comparison of maximum specific growth rates and lag times estimated from absorbance and viable count data by different mathematical models. J. Microbiol. Methods 43 (3), 183-196 (2001)

Gibson, A.M., N. Bratchell y T.A. Roberts. The effect of sodium chloride and temperature on the rate and extent of growth of Clostridium botulinum type $A$ in pausterized pork slurry. J. Appl. Bacteriol. 62 (6), 479490 (1987).

Grijspeerdt, K. y P. Vanrolleghem. Estimating the parameters of the Baranyi model for bacterial growth. Food Microbiol. 16 (6), 593-605 (1999).

Hills, B.P. y K.M. Wrigth. A new model for bacterial growth in heterogeneous system. J. Theor. Biol. 168 (1), 593-605 (1994).

McKellar, R.C., A heterogeneous population model the analysis of bacterial growth kinetics. Int. J. Food Microbiol. 36 (2-3), 179-186 (1997).

McMeekin, T.A., J.N. Olley, T. Ross y D.A. Ratkowsky. Predictive Microbiology: Theory and Application, 61-70. Research Studies Press Ltd., Tounton, Inglaterra (1993).

Perni, S., P.W. Andrew y G. Shama. Estimating of maximum growth rate from microbial growth curves: definition is everything. Food Microbiol. 22 (6), 491-495 (2005).

Poschet, F. y otros cinco autores. Sensivity analysis of microbial growth parameters distributions with respect of data quality and quantity by using Monte Carlo analysis. Math. Comput. Simul. 65 (3), 231-243 (2004).

Swinnen, I.A.M., K. Bernaerts, E.J.J. Dens, A.H. Geeraerd y J.F. Van Impe. Predictive modeling of the microbial lag phase: a review. Int. J. Food Microbiol. 94 (2), 137-159 (2004).

Zwietering, M.H., I. Jongenburger, F.M. Rombouts y K. Van't Riet. Modeling of the bacterial growth curve. Appl. Environ. Microbiol. 56 (6), 1875-1881 (1990). 
